\title{
Comportamento dos teores de sais em perfil de solo com vegetação nativa e cultura irrigadas
}

\author{
Behavior of salts content in soil profile with native vegetation and culture irrigated \\ ${ }^{1}$ Felipe Guedes de Souza, ${ }^{2}$ Soahd Arruda Rached Farias, ${ }^{3}$ José Geraldo de Araújo Ferreira Filho, ${ }^{4}$ Kyonelly Queila Duarte \\ Brito
}

\begin{abstract}
Resumo: A salinização do solo é um problema que vem crescendo em todo o mundo; principalmente nas regiões áridas e semiáridas, tendo um agravante maior devido a baixa precipitação e a elevada evaporação. Nestas condições nem sempre as aguas utilizadas são de boa qualidade afetando a capacidade das plantas de expressar seu máximo potencial produtivo. Diante disto, objetivou-se com o presente estudo avaliar o comportamento dos sais no solo sob condições naturais e irrigadas por águas residuárias da cidade de Campina Grande, PB durante o período de maio a julho de 2014. A pesquisa em campo foi realizada na propriedade Cajá que são abastecidas pelas águas do rio Bodocongó, destinada à produção de capim Mombaça, capim Tifton, Sorgo e Milho irrigados através de sistemas de irrigação como pivô, aspersão fixa, e inundação. Foram coletados três pontos distintos de amostras de solo nas profundidade de $10 \mathrm{~cm}, 30 \mathrm{~cm}, 60 \mathrm{~cm}, 75 \mathrm{~cm}, 90 \mathrm{~cm}$. Sendo o primeiro na área irrigada por superficial o segundo área nativo na propriedade vizinha e o terceiro na área irrigada do pivô. $\mathrm{O}$ aumento do $\mathrm{pH}$ nas áreas irrigadas tornando os solos alcalinos, em relação a salinidade verificou-se um aumento significante nos solos irrigados em decorrência dessa própria irrigação com aguas salinas e o PSI teve um aumento em decorrer da profundidade em todas as cinco amostras.
\end{abstract}

Palavras-chave: Salinidade, Pivô Central, Capim Tfiton.

Abstract: Soil salinization is a problem that has been growing throughout the world; especially in arid and semi-arid regions, and more aggravating because of low rainfall and high evaporation. Under these conditions the waters used are not always good quality affecting the ability of plants to express their full productive potential. In view of this, the aim of the present study was to evaluate the behavior of salts in the soil under natural conditions and irrigated by wastewater from the city of Campina Grande, PB during the period from May to July 2014. The field research was carried out on the property caja that are supplied by the waters of the river Bodocongó, for the production of grass Mombasa grass Tifton, Sorghum and corn irrigated by irrigation systems as pivot, fixed sprinkler and flood. We collected three distinct points of soil samples in the depth of $10 \mathrm{~cm}$, $30 \mathrm{~cm}, 60 \mathrm{~cm}, 75 \mathrm{~cm}, 90 \mathrm{~cm}$. It is the first in the area irrigated by surface the second native area in the neighboring property and the third in the pivot irrigated area. The $\mathrm{pH}$ increase in irrigated areas making alkaline soils, in relation salinity there was a significant increase in irrigated soils due to this own irrigation with saline water and PSI was increased in the course of the depth in all five samples.

Key words: Salinity, Central Pivot, Capin Tfiton.

\footnotetext{
*Autor para correspondência

Recebido para publicação em 15/02/2016; aprovado em 10/06/2016

${ }^{1}$ Mestrando em Engenharia Agrícola, Universidade Federal de Campina Grande, Campina Grande, felipeguedes.eng@gmail.com, (83) 996721083,

${ }^{2}$ Doutora em engenharia agrícola, Universidade Federal de Campina Grande, soahd@ deag.ufcg.edu.br,

${ }^{3}$ Engenheiro Agrícola, Universidade Federal de Campina Grande, ferreiraa.filho@ gmail.com,

${ }^{4}$ Mestre em engenharia agrícola, Universidade Federal de Campina Grande, queiladuarte@ hotmail.com.
} 


\section{INTRODUÇÃO}

A agricultura irrigada depende tanto da quantidade como da qualidade da água, no entanto, o aspecto da qualidade tem sido desprezado devido ao fato de que, no passado, em geral as fontes de água, eram abundantes, de boa qualidade e de fácil utilização, esta situação está se alterando em muitos lugares. O uso intensivo de praticamente todas as águas de boa qualidade implica que, tanto nos projetos novos como nos antigos que requerem águas adicionais, tem-se que recorrer às águas de qualidade inferior. Para evitar problemas consequentes, deve-se existir planejamento efetivo que assegure melhor uso possível das águas, de acordo com a qualidade (AYERS ; WESTCOT, 1999).

A salinização do solo é um problema que vem crescendo em todo o mundo; acredita-se que cerca de bilhões de hectares de solos sejam alterados por sais, com grande parte de todas as áreas irrigadas do mundo sofrendo com a redução da produção em decorrência do excesso de sais.

Tais solos são principalmente encontrados em climas áridos e semiáridos e ocorrem em mais de 100 países em todos os continentes, com exceção da Antártica. No Brasil solos salinos e sódicos ocorrem no Rio Grande do Sul, na região do Pantanal Mato-grossense e, com predomínio na região semiárida do Nordeste (RIBEIRO et al., 2003). Além de se caracterizar como um grave problema ambiental, a elevada concentração de sais no solo causa perdas consideráveis para agricultura mundial ou por causar perdas em cultivos já estabelecidos ou por inviabilizar a exploração de novas áreas agricultáveis (FLOWERS, 2004; MUNNS et al., 2006).

O processo de salinização envolve a concentração de sais solúveis na solução do solo e resulta na formação dos solos salinos, que resulta da acumulação de sais solúveis de $\mathrm{Na}^{+}, \mathrm{Ca}^{2+}, \mathrm{Mg}^{2+}$ e $\mathrm{K}^{+}$nos horizontes do solo. Ressaltando que, os principais sais encontrados são: cloretos e sulfatos de

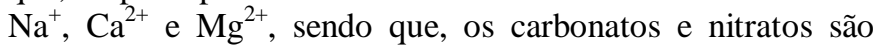
encontrados em quantidades reduzidas. Como destaque deve ser lembrado que em razão do excesso de sais e da ausência de quantidades relevantes de $\mathrm{Na}$, os solos salinos são floculados e não apresentam limitação, devido a permeabilidade. Os solos salino-sódicos são formados quando a Percentagem de Sódio Trocável (PST) atinge valores maiores ou iguais a $15 \%$, e os níveis de salinidade permanecem altos, com uma condutividade elétrica $(\mathrm{CE}) \geq 4$ $\mathrm{dS} \cdot \mathrm{m}^{-1}$. O processo de solonização promove a formação de solos sódicos, e é constituído por dois subprocessos: sodificação e dessalinização. A sodificação, primeira etapa, é o processo de passagem do íon $\mathrm{Na}^{+}$da solução do solo para o complexo de troca, formando os solos denominados de salinosódicos, enquanto a dessalinização, etapa final, promove a lavagem dos sais solúveis, resultando na formação de solos unicamente sódicos (RIBEIRO et al., 2003; RIBEIRO, 2010).

Devido a seu efeito sobre a permeabilidade do solo e sobre a nutrição e toxicidade das plantas a sodicidade é utilizado na classificação da água de irrigação (ALMEIDA, 2010). Barreto et al., (2012), acrescenta que o pH é um importante indicador das condições química do solo, por possuir capacidade de interferir na disposição de vários elementos químicos, essenciais ao desenvolvimento vegetal e a CE como indicador da salinidade no solo.
Águas residuárias são provenientes de esgotos domésticos e industriais. Os primeiros, também chamados de sanitários, contêm cerca de 99,9\% de água, 0,01\% de sólidos orgânicos e inorgânicos e carreiam dejetos de origem humana, os quais podem conter microrganismos patogênicos. Assim, o lançamento de esgotos não tratados em corpos aquáticos são vetores de poluição e contaminação e responsáveis pela disseminação de doenças entéricas (ALJASSER, 2011). No Brasil, especificamente na região Nordeste, a utilização de águas residuárias se destaca como uma promissora técnica de convivência com a seca (SOUSA et al., 2006).

As maiores vantagens do aproveitamento da água residuária para fins agrícolas residem na conservação da água disponível e na possibilidade de aporte e reciclagem de nutrientes, reduzindo a necessidade de fertilizantes químicos e contribuindo para a preservação do meio ambiente (SIMÕES et al., 2013). O principal agravante na utilização de esgoto na agricultura é o aspecto sanitário, uma vez que os mesmos apresentam uma grande variedade de microrganismos patogênicos (PALESE et al., 2009).

O presente estudo objetivou avaliar o comportamento dos sais no solo sob condições naturais e irrigadas por águas residuárias da cidade de Campina Grande, PB durante o período de maio a julho de 2014.

\section{MATERIAL E MÉTODOS}

A pesquisa foi realizada nos meses de maio a julho de 2014 na propriedade Cajá, localizada no município de Campina Grande no bairro da Catingueira, cujas coordenadas geográficas são: $07^{\circ} 17^{\prime} 55^{\prime}$ ' latitude Sul e $35^{\circ} 56^{\prime} 27^{\prime}$, longitude Oeste.

A propriedade é abastecida pelas águas do Riacho Bodocongó, caracterizadas como águas residuárias, onde essa água é utilizada na produção de capim Tifton, Sorgo e Milho. Essas culturas são irrigadas através de dois sistemas de irrigação são eles: pivô, superfície por inundação.

Para a caracterização do perfil de solo foram coletadas amostras de solo em três áreas de produção agrícola distinta, mas com características semelhantes. Foi feita uma abertura no perfil do solo e retirado parcelas de solos nas profundidades de $10 \mathrm{~cm}, 30 \mathrm{~cm}, 60 \mathrm{~cm}, 75 \mathrm{~cm}$ e $90 \mathrm{~cm}$.

$\mathrm{O}$ primeiro ponto foi perfurado na área de cultivo de milho e sorgo irrigado a 2 anos por um pivô central com as águas do Riacho Bodocongó. A perfuração do segundo ponto foi realizada em uma área vizinha a propriedade, sendo caracterizado como uma área nativa sem utilização na agricultura. $\mathrm{O}$ último ponto de coleta foi nas proximidades do galpão da fazenda, sendo cultivado nesta área o capim Tifton e irrigado por inundação há 10 anos com as águas do Riacho de Bodocongó.

Foi coletada amostra de água do riacho Bodocongó e encaminhada para análise no laboratório de Irrigação e Salinidade (LIS) da Unidade Acadêmica de Engenharia Agrícola da Universidade Federal de Campina Grande, onde analisou-se a condutividade elétrica (CEes) e o pH o da amostra de água.

Os dados coletados das três áreas foram analisados através de uma estatística descritiva (mínimo, máximo, média, mediana, desvio padrão, coeficiente de variação) como forma de obter a predominância de tendência das amostras e produzir um perfil de deslocamento de alguns sais ao longo 
da profundidade comparando as duas áreas irrigadas com a área nativa.

A classificação dos solos quanto à sodicidade foi realizada de acordo com os critérios propostos por Pizarro, 1985; Cabello, (1996),

\section{RESULTADOS E DISCUSSÃO}

$\mathrm{Na}$ tabela 1 apresentam-se os parâmetros de classificação do solo quanto a salinidade, a sodicidade, como a classe do solo para as três áreas da propriedade foram determinados no Laboratório de Irrigação e Salinidade (LIS) da Universidade Federal de Campina Grande.

Observa-se na tabela 1 que em relação à classificação de salinidade existe a predominância $90 \%$ de solos ligeiramente salinos naqueles que estão sendo irrigados com a água do riacho de Bodocongó já em relação ao solo sem atividade agrícola, houve uma predominância de solo não salino nas primeiras camadas até $60 \mathrm{~cm}$ já nas duas últimas camadas pode-se observar que tiveram um comportamento de ligeiramente salino. Em relação a sodicidade apresenta-se uma predominância de solos mediamente sódicos nas duas camadas irrigadas influência da irrigação já em relação ao solo nativo verifica-se uma normalidade a sodicidade. Por fim a classe do solo foi tida como normal na maioria das amostras de solo.

Na tabela 2 apresenta-se a classificação dos solos quanto à classe textural, nota-se uma predominância de $66,6 \%$ da classe de solos franco arenosos nos perfis até $60 \mathrm{~cm}$ de profundidades. Nos solos nativos, a partir dos $75 \mathrm{~cm}$ de profundidade há ocorrência de solos um pouco mais argilosos com relação às profundidades anteriores. Para os solos irrigados de maneira superficial percebe-se exclusivamente a presença de solos da classe areia.

Uma das principais características dos solos solonedz solodizado, é a camada superficial ácida ou ligeiramente ácida, o que pode ser observado nas análises do solo nativo, a alcalinidade e os sais presente na água do riacho de Bodocongó, podem ter ocasionado um aumento no $\mathrm{pH}$ do solo analisado.

O comportamento de sais (condutividade elétrica do estrato saturado) (CEes) é percebido coerente pelo tempo de uso de aguas irrigadas com teores elevados de sais do rio Bodocongó que influenciou os solos irrigados que tiveram sua CEes elevadas consideravelmente em comparação com o solo nativo, a superfície fica com mais sais, pela influência da evaporação e a concentração de sais no final devido o deslocamento ao longo das irrigações para pontos mais baixos, e que possui difícil lixiviação decorrente do teor de argila presente nas ultimas camadas dos solos nativos e irrigados por pivô, como no perfil de inundação tem pouca argila, o teor de sais foi bem mais presente, até pela excessiva quantidade de água que pode ser aplicada pela falta de controle de lamina. Pois o capim Tifton que é cultivado nessa área é irrigado através de tubos que lança a água sobre a superfície de forma descontrolada.

Tabela 1 - Classificação da Salinidade, Sodicidade e Classe do solo

\begin{tabular}{|c|c|c|c|c|}
\hline Áreas & Profundidade $(\mathrm{cm})$ & Salinidade & Sodicidade & Classe do solo \\
\hline \multirow{5}{*}{ Solo Irrigado Pivô } & 10 & Lig. Salino & Med. Sódico & Normal \\
\hline & 30 & Lig. Salino & Med. Sódico & Sódico \\
\hline & 60 & Lig. Salino & Med. Sódico & Normal \\
\hline & 75 & Lig. Salino & Med. Sódico & Normal \\
\hline & 90 & (Rocha) & - & - \\
\hline \multirow{5}{*}{ Solo Nativo } & 10 & Não Salino & Normal & Normal \\
\hline & 30 & Não Salino & Normal & Normal \\
\hline & 60 & Não Salino & Normal & Normal \\
\hline & 75 & Lig. Salino & Lig. Sódico & Normal \\
\hline & 90 & Lig. Salino & Med. Sódico & Sódico \\
\hline \multirow{5}{*}{ Solo Irrigado Superficial } & 10 & Med. Salino & Med. Sódico & Salino \\
\hline & 30 & Lig. Salino & Med. Sódico & Sódico \\
\hline & 60 & Lig. Salino & Med. Sódico & Normal \\
\hline & 75 & Não Salino & Normal & Normal \\
\hline & 90 & Lig. Salino & Med. Sódico & Normal \\
\hline
\end{tabular}

Tabela 2 - Classificação de Textura do Solo

\begin{tabular}{ccc}
\hline Áreas & Profundidade $(\mathrm{cm})$ & Classificação Textural \\
\hline \multirow{3}{*}{ Solo Irrigado Pivô } & 10 & Franco Arenoso \\
& 30 & Franco Arenoso \\
& 60 & Franco Arenoso \\
& 75 & Franco Arg. Arenoso \\
& 90 & (Rocha) \\
\hline \multirow{2}{*}{ Solo Nativo } & 10 & Franco Arenoso \\
& 30 & Franco Arenoso \\
& 60 & Franco Arenoso \\
& 75 & Argila Arenoso \\
& 90 & Franco Arg. Arenoso \\
\hline \multirow{3}{*}{ Solo Irrigado Superficial } & 10 & Areia Franca \\
& 30 & Areia Franca \\
& 60 & Areia Franca \\
& 75 & Areia Franca \\
& 90 & Areia Franca
\end{tabular}


As Figuras 1 e 2 apresentam os resultados de $\mathrm{pH}$ e condutividade elétrica, para os solos estudados, em função das profundidades analisadas. De acordo com os valores mínimos $(4,67)$ e máximo $(8,03)$ de $\mathrm{pH}$, verifica-se que na região analisada as amostras de solos estão variando de moderadamente ácida a alcalina de acordo com os critérios adotados por RICHARDS 1954. Com base nos dados de pH partir das análises, pode-se observar que a irrigação, tanto por pivô quanto por superficial, está tornando os solos que recebem esta irrigação alcalinos.

Apresentados no gráfico 2, a qual indica os níveis de sais no solo, pode-se afirmar que as amostras foram consideradas entre ligeiramente salinos a médio salinos tendo como valores mínimos $(0,36)$ e máximos $(5,60)$, sendo que os valores mais altos de salinidade foram encontrados no solo irrigados superficialmente nas primeiras camadas, porém existe uma tendência a partir da camada de $60 \mathrm{~cm}$ que os três perfis se torne salinos mesmo o solo nativo.

Figura 1: Relação entre $\mathrm{pH}$ do solo e Profundidade em cm

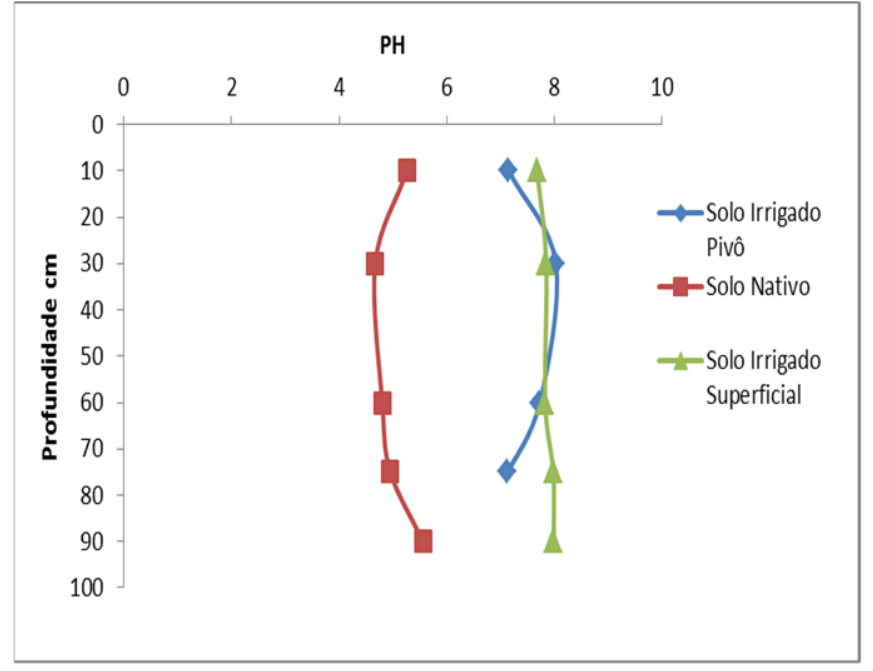

Figura 2: Relação entre CEes do solo e Profundidade em cm

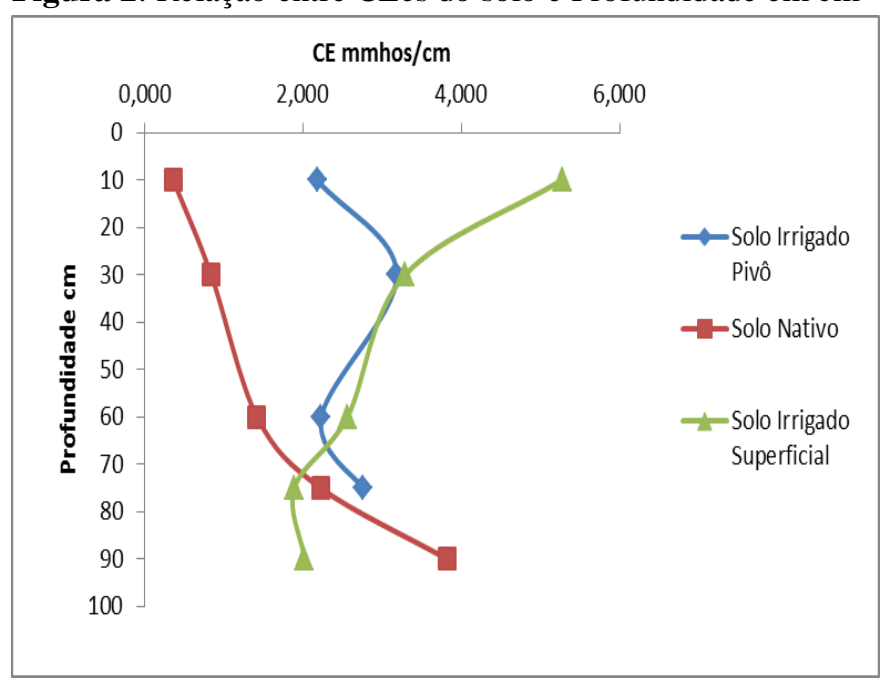

Observa-se na figura 3 que no solo nativo a quantidade de sódio mantem-se quase que constante nas primeiras camadas do solo, a partir dos $30 \mathrm{~cm}$ nota-se um acréscimo na concentração de sódio presente no solo, o que pode ter sido ocasionado devido a maior presença de areia nas camadas superficiais nas quais o sódio é facilmente lixiviado para as camadas mais inferiores onde há maior ocorrência de argila no solo, proporcionando ao sódio uma maior interação entre o sódio e a solução do solo. Ainda no referido gráfico, os solos irrigados, classe textural areia franca, as concentrações iniciais de sódio são maiores nas camadas iniciais, quando comparadas à concentração inicial do solo nativo. Corroborando com os resultados apresentados por Aguiar Netto et al. (2007), os quais verificaram que as maiores concentrações de de sódio ocorreram na camada superficial do solo $(0-0,2 \mathrm{~m})$, devido a falta de um manejo da irrigação adequado visando ao controle da salinidade.

A porcentagem de sódio trocável é apresentada na figura 4, verifica-se um aumento nos valores de PSI com o aumento da profundidade tanto no solo nativo, como nos solos irrigados, o que pode estar sendo ocasionado devido a um processo de lixiviação, seja por meio da irrigação ou através dos níveis de precipitação, levando os sais das camadas superficiais para as camadas mais inferiores do perfil do solo. Observa-se ainda na figura 4 que para os solos irrigados, os valores de PSI variam entre 10 e $15 \%$ na profundidade de $0-0,6 \mathrm{~m}$. O limite preconizado como o que define solos sódicos é de $15 \%$ de PST, limite este adotado pelo Sistema Brasileiro de Classificação de Solos (EMBRAPA, 2013). Freire et al. (2003) observaram que valores de PST inferiores a $15 \%$ já proporcionam degradação de solos.

O excesso de sais solúveis e/ou sódio trocável, que caracterizam os solos afetados por sais como salino, salinosódico ou sódico, dificulta a absorção de água do solo pelas plantas, induz à toxicidade de íons específicos (sódio e cloreto, principalmente), causa desequilíbrio nutricional e impede a infiltração de água no solo, provocando redução do crescimento e diminuição do rendimento das culturas. (HOLANDA et al., 2010).

Figura 3: Relação entre sódio e profundidade cm.

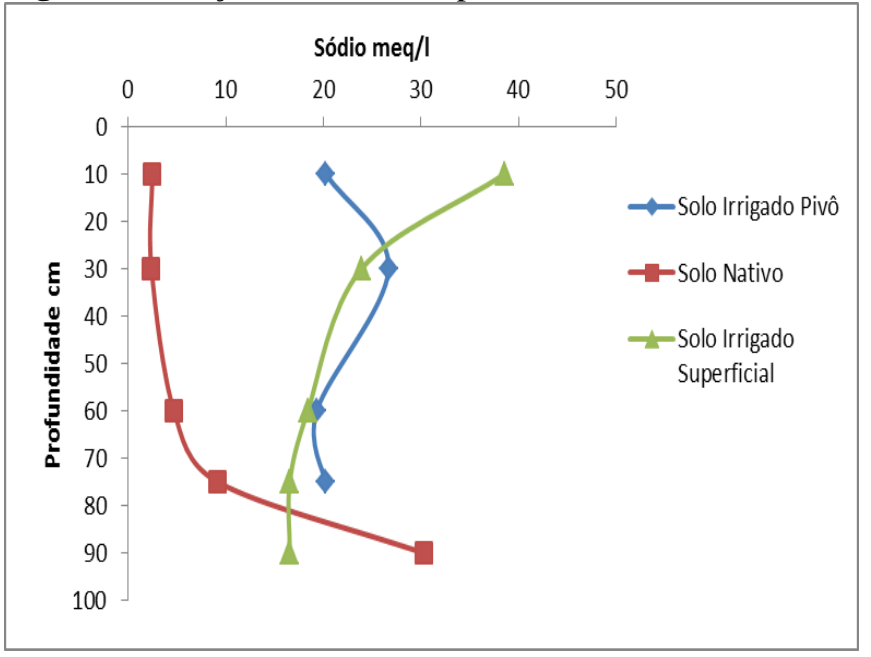


Figura 4 - Relação entre PSI e profundidade cm

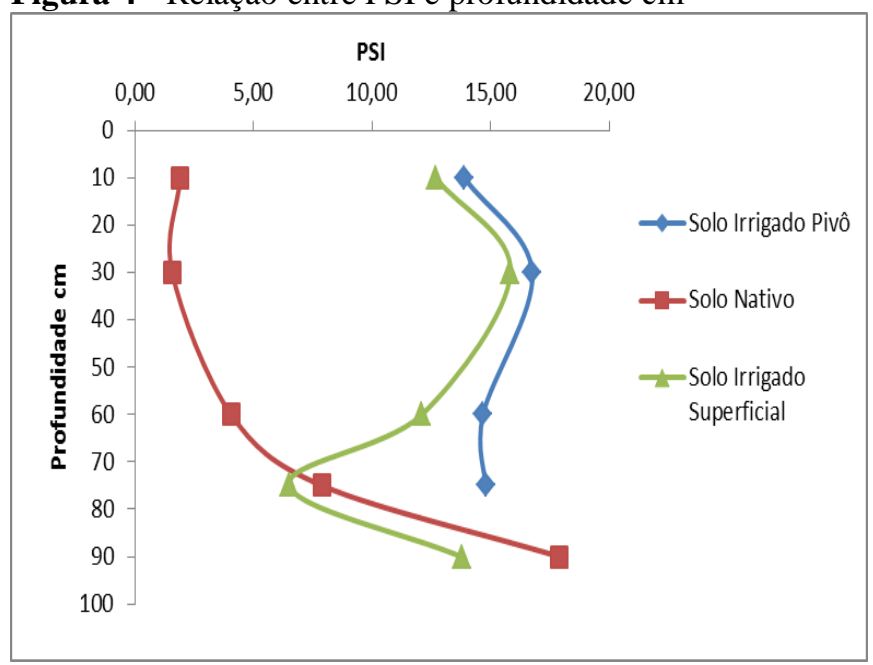

A figura 5 apresenta os valores em porcentagem da quantidade de argila no solo, para a área nativa o solo apresenta uma maior fração de argila na composição do solo, o que corrobora com a análise do solo para a mesma profundidade, tendo em vista que quanto maior a fração de argila no solo maior a capacidade do mesmo em reter grandes quantidades de cátions presentes na solução do solo, o que não ocorre para os solos irrigados devido à menor presença de argila em sua composição granulométrica.

Figura 5 - Relação entre \% argila e profundidade $\mathrm{cm}$.

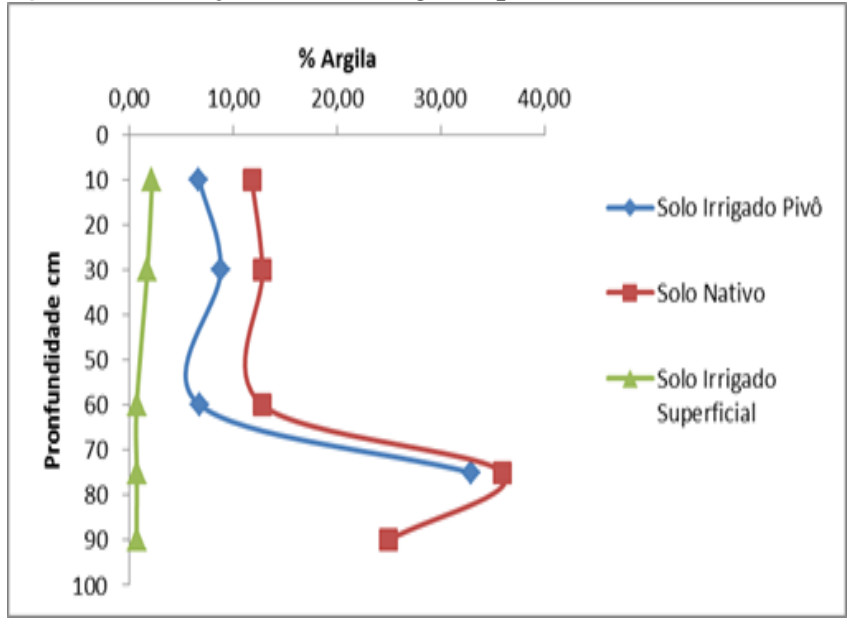

\section{CONCLUSÕES}

Em relação aos dados de pH partir das análises, pode-se observar que a irrigação, tanto por pivô quanto por superficial por inundação, está contribuindo para os solos que recebem esta irrigação tornarem-se alcalinos em comparação com o solo nativo onde ocorreu em todas as camadas predominância de um solo ácido.

Verificou-se um aumento nos valores do PSI conforme o aumento da profundidade tanto para o solo nativo como para os irrigados sendo ocasionado pelo processo de lixiviação dos sais tanto na forma de precipitação como pelo processo da irrigação e deficiência de drenagem.

A salinidade CEes teve uma variação de valores de 0,36 no solos nativos para 5,60 nas áreas que são irrigadas pelo Riacho de Bodocongó confirmando que a utilização de águas residuárias e com altos teores de sais de forma descontrolada é um fator de perturbação do solo.

\section{REFERÊNCIAS}

AL-JASSER, A. O. Saudi wastewater reuse standards for agricultural irrigation: Riyadh treatment plants effluent compliance. Journal of King Saud UniversityEngineering Sciences, v. 23, p. 1-8, 2011.

ALMEIDA, O. Á. DE: Qualidade da água de irrigação. Cruz das Almas : Embrapa Mandioca e Fruticultura, 2010. Disponível em <http://www.cnpmf.embrapa.br/publicacoes/livro_qualidade_ agua.pdf $>$ (acesso em outubro de 2012).

AYERS, R. S.; WESTCOT, D. W. A qualidade da água na agricultura. Campina Grande: UFPB, 1999. 153 p. (Estudos FAO: Irrigação e Drenagem, 29).

AGUiAR NETTO, A de O., GOMES, C. C. S., LINS, C. C. V., BARROS, A. C., CAMPECHE, L. F. S. M., BLANCO, F. F., Características químicas e salino-sodicidade dos solos do Perímetro Irrigado Califórnia, SE, Brasil. Ciência Rural, Santa Maria, v.37, n.6, p.1640-1645, 2007.

BARRETO, H. B. F.; AMARAL JÚNIOR, V. P. DO; MAIA, P. DE M. E.; SILVA NETO, S. J. DA: Variabilidade espacial do ph e condutividade elétrica em solo cultivado com arroz irrigado no Rio Grande do Norte. Revista Verde (Mossoró RN - Brasil) vol.7, no 1, pg. 182 - 188, jan/mar de 2012.

EMBRAPA - Empresa Brasileira de Pesquisa Agropecuária. Sistema Brasileiro de Classificação de Solos. Brasília: EMBRAPA, 2013. 353p.

FLOWERS, T. J. 2004. Improving crop salt tolerance. Journal of Experimental Botany, v.55, n.369, p.307-319

FREIRE, M. B. G. S., RUIZ, H. A., RIBEIRO, M. R., FERREIRA, P. A., ALVAREZ, V. H., FREIRE, F, J.: Condutividade hidráulica de solos de Pernambuco em resposta à condutividade elétrica e RAS da água de irrigação. Revista Brasileira de Engenharia Agrícola e Ambiental, v.7, p.45- 52, 2003.

HOLANDA, J. S., AMORIM, J. R. A., FEREIRA NETO, M., HOLANDA, A. C.: Qualidade da água para irrigação. In: GHEYI, H. R., DIAS, N. S., LACERDA, C. F.; Manejo da salinidade na agricultura: Estudo básico e aplicações. Fortaleza, INCT Sal, 2010. pg. 43-59.

MUNNS, R.; RICHARD, A. JAMES, R. A.; LAUCHLI, A. 2006. Approaches to increasing the salt tolerance of wheat and other cereals. Journal of Experimental Botany, v.57, n.5, p.1025-1043.

OLIVEIRA, L. B.; RIBEIRO, M. R.; FERRAZ, F. B.; JACOMINE, P. K. T. Classificação de solos planossólicos do sertão do Araripe. Revista Brasileira de Ciência do Solo, v.27, p.685-693, 2003. 
PALESE, A.; PASQUALE, V.; CELANO, G.; FIGLIUOLO, G.; MAISI, S.; XILOYANNIS, C. Irrigation of olive groves in Southern Italy with treated municipal wastewater: Effects on microbiological quality of soil and fruits. Agriculture, Ecosystems \& Environment, v. 129, p. 43-51, 2009.

PIZARRO CABELLO, F. Riegos localizados de alta frecuencia: goteo, microaspersión, exudación. 2. ed. Madrid: Ediciones Mundi-Prensa, 1996. 471 p.

RIBEIRO, M. R. 2010. Origem e Classificação dos Solos Afetados por Sais. In: GHEYI, H. R.; DIAS, N. S.; LACERDA, C. F. (Eds.). Manejo da Salinidade na Agricultura: Estudos Básicos e Aplicados. Fortaleza, INCTSal. p.11-19.

RIBEIRO, M. R.; FREIRE, F. J.; MONTENEGRO, A. A. A. 2003. Solos halomórficos no Brasil: Ocorrência, gênese, classificação, uso e manejo sustentável. In: CURI, N.; MARQUES, J. J.; GUILHERME, L. R. G.; LIMA, J. M.; LOPES, A. S; ALVAREZ, V. H. (eds.). Tópicos em Ciência do Solo. Viçosa: Sociedade Brasileira de Ciência do Solo, v.3, p.165-208.
RICHARDS, L. A. (ed): Diagnosis and Improvement of saline end alkali soils. Washington D. C.: United States Salinity Laboratory Staff. 166 pg., USDA. Agriculture Handbook nº 60, Issued February 1954.

SIMÕES, K.S.; PEIXOTO, M.F.S.P.; ALMEIDA, A.T.; LEDO, C.A.S.; PEIXOTO, C.P.; PEREIRA, F.A.C. Água residuária de esgoto doméstico tratado na atividade microbiana do solo e crescimento da mamoneira. Revista Brasileira de Engenharia Agrícola e Ambiental, v. 17, n.5, p. 518-523, 2013.

SOUSA; J.T.; HENRIQUE; I.N.; LEITE; V.D.; LOPES, W.S. Tratamento de águas residuárias: uma proposta para a sustentabilidade ambiental. Revista de Biologia e Ciências da Terra, v. 1, p. 90-97, 2006.

SOUTO, P. C.; SOUTO, J. S.; SANTOS, R. V.; ARAÚJO, G. T.; SOUTO, L. S. Decomposição de estercos dispostos em diferentes profundidades em área degradada no semiárido da Paraíba. Revista Brasileira de Ciência do Solo, v.29, p.125131. 